\section{MATHEMATICS AND PHYSICS AT THE BRITISH ASSOCIATION.}

THE address of the president of Section A, Prof. H. H. Turner, has been already printed in this journal (August 3r, p. 289).

\section{Cosmical Physics and Astronomy.}

The address was followed by a meteorological paper by Prof. W. J. Humphreys (of the U.S. Weather Bureau) on the earth as a radiator. Since our climates are not now perceptibly growing either colder or warmer, the total amount of heat received by the earth is substantially equal to the loss during the same time. But this statement does not apply to limited regions; and therefore to map the earth as a radiator it is necessary first to obtain temperature records within the isothermal region, where radiation alone is the controlling factor. From the experimental results the author deduces that the outward radiation is least near the equator, where the inward radiation is greatest, and greatest in temperate latitudes, a secondary minimum at the pole being indicated by the ascents in high latitudes. In the discussion it was pointed out that the results depend largely on the assumption that the atmosphere acts as a "grey" body. The values might be subject to considerable correction for irregularities in the absorbing power for different spectral regions. Dr. Shaw directed attention to the need for examining the influence of different thicknesses of water vapour in the atmosphere, and considered that reflection from cirrus clouds would introduce a considerable disturbing influence. A suggestion by Prof. Hicks that possibly the results were a consequence of the preponderating amount of ocean near the equator was met by Dr. Humphreys replying that the same results were obtained over ocean as over land.

Mr. L. Vegard, of Christiania, contributed a suggestive paper on the radiation producing aurora borealis, in which he starts from Birkeland's view that auroræ are caused by electric solar radiations, and endeavours to deduce the properties of these radiations. From the form and structure of the luminosity he infers that the electric radiations behave as though they are $\alpha$ rays. It is found, from the relation between range and velocity, that $\alpha$ rays will get down to heights varying between 70 and $300 \mathrm{~km}$., which agrees with observations. Further, calculations show that $\alpha$ rays will strike the atmosphere at an angular distance from the magnetic axis of about $17^{\circ}$, which gives the right position of the auroral zone. The equidistant bands so characteristic of the draperies he explains by assuming groups of homogeneous rays, starting under the same initial conditions, such as would be provided by radium and its disintegration products in the sun. The author made a strong case for his explanation of these interesting phenomena.

Dr. W. N. Shaw communicated an account of the thunderstorms of July 28 and 29. The London storm on July 28 was accompanied by a squall of wind which reached fifty-four miles an hour at South Kensington, and the storms on July 29, which occurred over nearly the whole of England and Ireland, were preceded by violent squalls which raised clouds of dust, particularly noticeable in South Wales. At Watlington (Mr. W. H. Dines) the disturbance occurred while the passage of a depression was in progress, and the barograph curve took an $\mathrm{M}$ form instead of the usual $\boldsymbol{N}^{-}$. The records of temperature, wind direction, and rainfall have still to be examined with the object of tracing the physical processes underlying the disturbance.

In the same department Commander Campbell Hepworth communicated a paper on the effect of the Labrador current upon the surface temperature of the North Atlantic, and of the latter upon the air temperature and barometric pressure over the British Isles. The purpose of the paper was to show the importance of the Labrador current in modifying the influence of the Gulf Stream.

Dr. Shaw showed models representing air currents up to heights of ro kilometres which had been obtained from the observations of pilot balloons made at Ditcham Park by Mr. Cave. The models showed some of the types of motion which occur, and were very instructive to those unfamiliar with the details of upper-air observations.
Dr. Humphreys read a paper on the water vapour in the atmosphere on clear days, a quantity of great importance in the determination of the solar constant. He found a value of 87 per cent. of the value found by Hann from observations made in all kinds of weather.

Mr. Gold gave a brief account of the results obtained from the ascents in Ireland, undertaken by the committee for the investigation of the upper atmosphere. Three successful ascents had been made from Mungret College, Limerick, in the present year, and on July 6 values for the temperature had been obtained up to $21 \mathrm{~km}$.

Dr. Dickson put forward the suggestion that the treatment of general atmospheric circulation might be simplified by taking the equatorial circulation to form a system by itself. It was pointed out in the discussion that general dynamical considerations rendered such an hypothesis untenable.

Mr. Craig read a paper by Dr. Ball and himself on the use of diagrams in the classification of climate. The diagrams dealt with temperature and humidity, and showed the annual course of these elements by a single closed figure for each of a selection of places from different parts of the world. The shape and orientation of the figure varied with the climate, and showed at once its principal features. Mr. Craig pointed out how they could be used not only by the man who was considering his health, but also in connection with such problems as cotton-growing.

Prof. J. Milne presented the sixteenth report of the committee on seismological investigations. Of the many interesting things in this report we may instance the observations on tidal load at Ryde in the Isle of Wight. By means of an instrument installed in a cellar in the Royal Victoria Yacht Club at Ryde and with its boom oriented east and west, a 10-foot tide in the Solent is found to produce an angular deflection of $0.85^{\prime \prime}$. At Bidston a ro-foot tide gives at a distance of two miles a tilt of $0.2^{\prime \prime}$. A curious feature of the Ryde photograms is the flatness of many of the crests and hollows of the deflections, which seems to indicate that from time to time the water remains high (or low) for several hours. When the boom was pointed north, or toward the advancing and retreating tide, the resulting photngrams were practically straight lines.

Prof. H. H. Turner read a note on the periodogram of earthquake frequency from seven to twenty years, in which he investigated by Schuster's methods the possibility of periodicities in earthquakes, making use of the data in the "Catalogue of Large Earthquakes" recently edited by Prof. Milne. Within the range of periods examined, the only chance of a real periodicity is one of nineteen years, suggesting a nutational effect depending on the moon's nodes.

A paper by Mr. F. Napier Denison, on horizontal pendulum movements in relation to certain phenomena, was read by Prof. Milne, who also read one on the solar cycle, the Jamaica rainfall, and earthquakes cycles, by Mr. Maxwell Hall, dealing with observations extending from 1870 to 1910. Excepting a rainfall minimum in 1875 , the minima for rainfall follow sun-spot maxima and minima by $1 \frac{1}{2}$ or 2 years. The rainfall maxima are more irregular. The earthquake maxima follow solar maxima by 2 years, while the minima follow solar minima by 2 or 3 years. Prof. O. Pettersson followed with a very interesting paper on parallactic tides set up in the bottom layers of the sea by the moon (see report of Section E).

\section{Stellar Distribution.}

The proceedings on Tuesday, September 5, commenced with a discussion on stellar distribution and movements, opened by Mr. A. S. Eddington, who stated that in attempt ing to form a conception of the structure of the universe as revealed by modern researches, we have to take into account the following principal phenomena. The great mass of the stars are distributed in a lens- or bun-shaped system, in which our sun occupies a nearly central position; round this, and in the same plane, are coiled the clusters, which make up the Milky Way. In the central parts of this the stars form two great streams moving in opposite directions; this is most easily explained as being the result of two more or less independent systems of stars No. 2โ89, VOL. 87 ] 
having become intermixed. We have also to take note of "moving clusters," that is, groups of stars widely separated in space, which have almost identical motions, and are thus clearly connected in their origin. A whole class of stars, those of the helium type of spectrum, are exceptional, as they do not show the phenomenon of the two star-streams, and have very little motion of any sort, individual or systematic. This is perhaps due to the fact that they are extremely remote, and lie beyond the region through which the star-streams prevail. Finally, there is the recently discovered connection between spectral type and linear motion; the later the type of spectrum (as regards evolutionary development) the greater the average speed of the stars.

By considering the stars known to have a parallax greater than $0 \cdot 2^{\prime \prime}$ (seventeen in number), some important results are illustrated, notably the sparsity with which stars are distributed in space, the very heavy proportion of binary systems, the very different degrees of intrinsic brightness of stars, and the fact that the relative frequency of the different spectral types as seen in the sky is utterly misleading as an indication of their abundance in space. The publication by Prof. Boss last year of more than 6000 welldetermined proper motions of stars distributed over the whole sky has greatly assisted investigations of the two star-streams. The bipolarity of the stellar motions is very clearly shown in the new data. Besides the theory of two star-drifts, an ellipsoidal theory, and more recently a threedrift theory, have been employed to represent the distribution of velocities. The theories have much in common, and it will be very difficult to distinguish between them. An attempt has been made to arrive at the law of velocities directly from the observations, without recourse to a particular theory; but the work, which involves an integral equation, has presented some difficulties, and is not yet completed.

The remarkable result that a star's motion appears to increase with its age, apparently implying that a star is born with practically no motion, leads us to inquire into the causes which produce stellar motions. From the consideration of the moving clusters it appears that the chance approaches of neighbouring stars have no appreciable effect, and the cause must be the resultant attraction of the whole mass of stars - a solution which, however, is not without its difficulties. Dr. Halm has suggested a law of equipartition of energy among the stars implying hat the dependence of velocity is not really on the spectral type, but on the mass of the star; it seems that the stars of later types are progressively less heavy than those of early type. A third possibility is that distance from the centre of the stellar system is the determining cause. The hypothesis has recently been revived that the stellar system forms a spiral nebula similar to the thousands of spiral nebulæ in the sky. This theory, though highly speculative, appears to represent fairly well the observed distribution of the stars and the Milky. Way, and the double-branched spiral form presumably involves motion in two opposite directions in the centre of the system similar to that presented by the two star-streams.

During the subsequent discussion Prof. Turner exhibited a model of the Ursa Major " moving cluster," specially showing that the component stars were nearly in one plane. Mr. A. R. Hinks emphasised that at present we are only getting at the central parts, and we do not know what fraction this part bears to the whole. In reference to the bun-shaped system, he considered that absorption of light had not been taken account of as it should be. He thought the spiral nebula conception was unnecessarily grandiose; he did not think that the star clouds of the Milky Way were well represented by the arms of a spiral nebula, as the latter seemed generally to be gaseous. He questioned whether anyone had yet explained the dynamics of the spiral motion in a nebula. He considered it premature to assume that there were not more than seventeen stars with parallax greater than $0 \cdot 2^{\prime \prime}$. He also criticised various points in nomenclature; in particular, the term star-cluster was being used in two senses.

The Astronomer Royal" (Prof. F. W. Dyson) emphasised the value of the information given by measured parallaxes and the importance of making more determinations. The real difficulty is a lack of a knowledge of the actual distances

No. 2 I 89 , VOL. 87$]$ of stars. $\mathrm{He}$ thought that the best way was to try to explain the velocities of stars gravitationally, and alluded to Lord Kelvin's satisfactory attempt in this direction to connect the total mass of the stars with the observed velocities. Prof. Turner and Mr. F. Bellamy explained the work done at Oxford in determining large proper motions; these were (unlike the stars as a whole) distributed nearly uniformly round the sky, the slight excess being in directions at right angles to the Milky Way. Father Cortie asked if there was not a contradiction in the fact the.t whereas the later-type stars had the larger velocities, yet they were relatively more abundant in the slowmoving drift than in the fast one. Mr. S. Stratton pointed out the anomalous position of the planetary nebulæ; their large velocities would place them at the end as the last stage of evolution. Mr. H. Hilton asked whether the velocity would not be least at the centre of a system of stars and increase outwards.

Mr. Eddington, replying to Prof. Turner, explained that the excess with large proper motions at right angles to the Milky Way was due to the solar motion coming in with greatest effect there in the Oxford zone. Replying to $\mathrm{Mr}$. Hilton, he explained that he had regarded the stars not as moving in orbits, but as moving from outside to inside, and vice versa. Replying to Father Cortie, he explained that it is the individual motions of later-type stars that are greatest, as distinct from the drift motions.

Father Cortie and Mr. J. H. Worthington gave some particulars in regard to endeavours to view the recent eclipse.

The programme for Wednesday, September 6, contained two papers by Mr. S. Stratton, which in his enforced absence had to be taken as read. One of these, on an unusual meteor observed at Portsmouth on August 3I, described a meteor with an apparent path in the form of a letter $\mathbf{J}$, which probably arose from foreshortening accompanied by swerve. The swerve might be traced to spin and resistance or to unequal heating effects. The second paper, on the possible relations between sun-spots and the planets, contained a discussion of sun-spot material tabulated to show phase effects due to Venus and Jupiter. The conclusion is against the validity of conclusions come to recently by Prof. Schuster in connection with the influence of Venus and Mercury. A longer period of observation is apparently necessary before trustworthy results can be obtained.

\section{Mathematics.}

In the department of mathematics Lieut.-Colonel Allan Cunningham, R.E., read a paper on Mersenne's numbers. These numbers are of the form $\mathrm{M}_{q}=2^{q}-\mathrm{I}$, where $q$ is a prime number. Since the appearance of Mr. W. W. Rouse Ball's paper in 1892 Prof. F. N. Cole has factorised $M_{67}$ Lieut.-Colonel Allan Cunningham has factorised $\mathbf{M}^{67}$, $M_{163}$, and $M_{197}$, while $H$. J. Woodall has recently factorised $M_{181}^{163}$. This leaves still unverified (as composite) only fifteen out of the forty-four numbers (with $q<257$ ) originally affirmed by Mersenne to be composite, viz. when

$q=101,103,107,109,137,139,149,157,167,173,193$, $199,227,229,24 \mathrm{I}$

A complete list of all the possible divisors $<\mathrm{I}$ million of these fifteen numbers has been prepared by $\mathrm{Mr}$. A. Gérardin (of Nancy, France) and Lieut.-Colonel Allan Cunningham, working independently. These trial divisors have been tested by the latter up to 500,000 without success (every "trial divisor" was tried twice).

Prof. J. C. Fields read a paper on relations between the double points and branch points of a plane algebraic curve $\mathrm{F}(x, y)=y^{n}+\mathrm{F}_{n-1} y^{n-1}+$, \&c., which presents no singularities at infinity and the finite point singularities of which consist of nodes and ordinary cusps. On representing an arbitrary polynomial in $(x, y)$ of degree $n-2$ by the notation $\mathrm{G}(x, y)=\Sigma_{r_{3}} d_{r_{s}} x^{r} y^{s}$ a simple proof was given of the formula $\Sigma_{\lambda} c_{\lambda} \frac{G\left(a_{\lambda}, b_{\lambda}\right)}{F_{b_{\lambda}}^{\prime \prime}\left(a_{\lambda} b_{\lambda}\right)}=d_{o},{ }_{n-2}$ where the summation is extended to all points $\left(a_{\lambda}, b_{\lambda}\right)$ which are ordinary branch points, nodes, or cusps of the curve, the coefficient $c_{\lambda}$ having as value either $\mathrm{I}, 2$, or 3 , according as the corresponding point is an ordinary branch point, a node, or a cusp. 
Mr. H. Bateman then contributed a note on the transformation of an electromagnetic field into itself. It is hoped that a discussion of the infinitesimal transformations will lead to equations of motion which will complete the electromagnetic scheme. Following the work of Mr. Hargreaves on the effect of an infinitesimal transformation on certain integral forms, it is assumed that these integral forms are invariants for the infinitesimal transformation. The analysis then indicates that two forms are exact differentials. Some types of infinitesimal transformations satisfying the conditions were obtained for particular electromagnetic fields, and the transformations were interpreted geometrically.

An account of the report of the committee for the further tabulation of Bessel and other functions was given by $\mathrm{Mr}$. Nicholson. In the report tables are given (calculated by Mr. J. R. Airey) of the Neumann functions $\mathrm{G}_{n}(x)$ and $Y_{n}(x)$ to seven decimal places for $n=0$ and $n=\mathrm{I}$, and for values of the argument from $0 \cdot x$ to 16.0 by intervals of o.I. During the course of the year Sir G. Greenhill has brought forward a scheme for the rearrangement of the elliptic functions tables on a new basis. This scheme has now received the approval of the association, and a grant has been made towards the expenses of computation.

In this department $\mathrm{Mr}$. H. Bateman contributed a paper on a geometrical theorem connected with six lines in space. $\mathrm{PP}^{\prime}, \mathrm{QQ}^{\prime}, \mathrm{RR}^{\prime}$ are three pairs of lines in space. $L^{\prime}$ are the common transversals of $Q^{\prime}, R^{\prime}$ $\mathrm{MM}^{\prime}$ the cominon transversals of $\mathrm{RR}^{\prime}, \mathrm{PP}^{\prime}$; and $\mathrm{NN}^{\prime}$ the common transversals of $\mathrm{PP}^{\prime}, \mathrm{QQ}^{\prime}$. If $\mathrm{QQ}^{\prime} \mathrm{RR}^{\prime}$ belong to a regulus, then $\mathrm{MM}^{\prime} \mathrm{NN}^{\prime}$ also belong to a regulus.

Mr. H. Hilton read a paper on the canonical form of an orthogonal substitution. After pointing out a short method of reducing a real orthogonal substitution to a canonical form, he discussed the analogous problem for an orthogonal substitution with complex coefficients. A canonical form was obtained in which the linear equation of the substitution were arranged in blocks, some of which contained two and others only one member. The leadins coefficient occurring in one type of block was the reciprocal of the corresponding coefficient occurring in an associated block.

Prof. J. C. Fields read a paper on proof of certain theorems relating to adjoint orders of coincidence, viz. :(I) In the reduced form of a rational function of $(z, u)$, which is adjoint for the value $z=a$ (or $z=\infty$ ), the coefficient of $u^{n-1}$ is integral with regard to the elements $z-a$ (or $\frac{I}{2}$ ). (2) If a rational function is adjoint for the value $z=\infty$, the degree of its reduced form is $z \mathrm{~N}-\mathrm{r}$. (3) The reduced form of a rational function adjoint for the value $z=a$ is integral with regard to the element $z-a$ if the equation $f(z, u)=\left(u-\mathrm{P}_{1}\right) \ldots\left(u-\mathrm{P}_{n}\right)=0$ (where $\mathrm{P}_{1} \ldots \mathrm{P}_{n}$ are power-series in an element $z-a$ (or $\frac{\mathrm{I}}{z}$ ) with exponents integral or fractional) is integral with regard to this element.

\section{The Principle of Relativity.}

The proceedings on Friday, September r, opened with a discussion on the principle of relativity, led by Mr. E. Cunningham, who pointed out that the scope of the hypothesis of relativity is exactly coincident in extent with its scope in Newtonian dynamics. The acceleration of a point is not physically indeterminate as its velocity is. The theory of relativity is, for example, quite consistent with the magnetic effects apparent to terrestrial observers being explained as arising from the rotation of the earth with a nearly stationary distribution of charge. Within the limits indicated above and within the realm of phenomena in which the sole determinative laws are those of the electron theory, the hypothesis becomes a mathematically demonstrable fact in the sense that it is not possible to choose a unique frame of reference for which alone the laws will hold good. Mr. Cunningham then sketched the transformations connecting the measurements made according to two frames of reference, each of which is equally adequate to express known phenomena, and explained what deductions can be drawn.

In the subsequent discussion Dr. W. F. G. Swann pointed out that the compliance of a system with the electromagnetic scheme is by no means a sufficient criterion for the possibility of its existence; for instance, a system of two singularities, moving along at a constant distance apart, with the field at each point the sum of the ordinary fields due to the separate motions, is a system in accordance with the scheme, but it is one impossible of existence in practice. The explanation of the impossibility of the existence of such systems is to be found in the fact that they could never evolve out of any actually existing system. In fact, if we take the electromagnetic scheme as complete, those uniformities in nature which we call "laws" are to be looked upon as due in part to the compliance of the universe with the scheme and in part to the individuality of the initial system started. A complete knowledge of the field at every point in space, both inside and outside the molecules, would, in conjunction with the electromagnetic scheme, theoretically give us all that we should need to ascertain both the past and subsequent history of the universe. Since we cannot know the complete field at some instant, we are driven to make up for our incomplete knowledge by formulating certain "subsidiary laws," such as laws involving the conception of forces between the singularities, electrical surface conditions, gravitation, \&c., the function of these laws being to restrict those systems which satisfy the scheme, and are also to be considered as possible of existence, to those which can spontaneously evolve out of the actually existing universe.

$\mathrm{Mr}$. H. Bateman emphasised the mathematical interest attached to the principle, because it unites several branches of mathematics, such as geometry, partial differential equations, generalised vector analysis, continuous groups of transformations, differential and integral invariants, \&c. $\mathrm{He}$ pointed out that there are two different types of transformations which can be used to transform one mathematical specification of an electromagnetic field into another. Those transformations which depend upon the electromagnetic fields which they can be used to transform form a much wider class than the spherical wave transformations, which can be applied to any electromagnetic field. The new transformations provide us with some very interesting analogues of mental phenomena.

Prof. F. Zeeman stated that the value of Fresnel's coefficient is easily deduced by means of the principle of relativity provided that no account is taken of dispersion. In that case the results of Fizeau's and Michelson and Morley's experiments on the propagation of light in flowing water agree with theory. The agreement is not so good if a dispersion term formally calculated by Lorentz be introduced. He asked whether the correction term would be the same if the principle of relativity were applied from the beginning to a dispersive medium. Prof. $\mathrm{G}$. $\mathrm{N}$. Lewis outlined some of the views which he has recently expressed in full in The Philosophical Magazine. Prof. A. E. H. Love warned one to be on the alert, because in the factor $K=\left(1-\beta^{2}\right)^{-\frac{1}{2}}$ we may be dealing only with first approximation. Secondly, he suggested that it was conceivable that terrestrial magnetism might be only an apparent phenomenon due to the rotation of the earth. $\mathrm{He}$ had, however, tested whether by taking rotatory axes one could obtain effects of the magnitude of terrestrial magnetism; but they turn out to be very small, and it seems hopeless to think of magnetism as due solely to the rotation.

Dr. C. V. Burton, after expressing his satisfaction that none had confessed a disbelief in the æther, urged the importance of a search for residual phenomena not falling within the electromagnetic scheme. Conceivably gravitation is such a phenomenon. There is, further, a question as to whether neighbouring electrically neutral masses exert forces upon one another in virtue of their motion through the æther. Such forces would be non-electromagnetic; experiments designed to detect them are in progress, but have so far given a null result. The entire absence of such forces would imply that matter takes up no room (positive or negative) in the æther.

So much time had been taken up by the discussion that none was left to $\mathrm{Mr}$. Cunningham to reply adequately to the numerous points raised. His scholarly paper introducing the discussion has been ordered to be printed in extenso; we look forward to perusing it in its extended form, and no doubt the answers to many of the questions will appear therein.

$$
\text { No. } 2 \text { I89, VOL. 87] }
$$


General Physics.

In the department of general physics Mr. N. E. Dorsey gave an account of the work done at the Bureau of Standards on the absolute measurement of electric current. The measurements were made with a balance of the Rayleigh type; the coils were wound bifilarly, of enamelinsulated wire, upon brass forms. A novel feature of the fixed coils is the provision, in the forms and back of the windings, of a channel, through which water can be pumped so as to maintain the coils at a constant temperature. A double-walled jacket with water circulation surrounded the movable coils for the same purpose. The mean value obtained of the electromotive force of the mean Weston normal cell (as defined at the Washington conference) at $20^{\circ} \mathrm{C}$. in terms of the international ohm and the Bureau balances is $1 \cdot 0 r 822_{4}$ (with a mean deviation of $\left.9 \times \mathrm{rO}^{-6}\right)$, which is 4 in 100 , 000 higher than the value obtained at the National Physical Laboratory. The cause of the discrepancy is not settled.

Prof. F. T. Trouton followed with a paper on peculiarities in the adsorption of salts by silica. Starting with very dilute solutions and gradually increasing their strengths, the amount of salt adsorbed first increases fast, then more slowly (or even decreases), then more quickly again. The thickness of the layer which exhibits the anomaly is calculated as being comparable with the usually given value for the range of molecular forces.

Dr. J. W. Nicholson contributed a paper on the atomic structure of the elements, with theoretical determinations of their atomic weights, in which an attempt was made to build up all the elementary atoms out of four protyles containing, respectively, $2,3,4$, and 5 electrons in a volume distribution of positive electricity. Representing the protyles by the symbols $\mathrm{Cn}$ (coronium), $\mathrm{H}$ (hydrogen), Nu (nebulium), Pf (protofluorine), the accompanying table indicates the deductions of the author with regard to the composition of several elements, allowance being made for the mass both of the positive and negative electrons.

\begin{tabular}{|c|c|c|c|c|c|}
\hline & & & Foum & Atom & eight \\
\hline & & & Formula & Calc. & Obs. \\
\hline $\begin{array}{l}\text { Helium } \\
\text { Argon } \\
\text { Krypton } \\
\text { Xenon } \\
\text { Neon ... }\end{array}$ & $\begin{array}{l}\ldots \\
\cdots \\
\cdots \\
\cdots \\
\cdots\end{array}$ & $\begin{array}{l}\cdots \\
\cdots \\
. . \\
\cdots \\
\cdots\end{array}$ & $\begin{array}{c}\text { Nu.Pf. } \\
5 \mathrm{He}_{2} \\
5\left\{\mathrm{Nu}_{4}(\mathrm{Pf} . \mathrm{H})_{3}\right\} \\
5\left\{\mathrm{He}_{4}(\mathrm{Pf} . \mathrm{HI})_{3}\right\} \\
2(\mathrm{Pf} . \mathrm{H})_{3}\end{array}$ & $\begin{array}{r}3 \cdot 99 \\
39 \cdot 88 \\
83 \cdot 0 \\
130 \cdot 29 \\
20 \cdot 21\end{array}$ & $\begin{array}{r}3 \cdot 99 \\
39 \cdot 88 \\
82 \cdot 9 \\
130 \cdot 2 \\
20 \cdot 2\end{array}$ \\
\hline
\end{tabular}

The coincidence between the calculated and observed values is very great, but the general attitude of those present seemed to be one of judicial pause pending the fuller presentation of the paper, stress being laid on the fact that any true scheme must ultimately give a satisfactory account of spectra. The rest of the morning was occupied with the reading of the reports of the committee for establishing a solar observatory in Australia and the committee on magnetic observations at Falmouth Observatory.

Prof. F. R. Watson contributed the results of experiments undertaken in connection with curing the echoes and reverberations in the auditorium at the University of Illinois. In accordance with theory, it is found that a broad sheet of warm air drawn over the head of the speaker and out at the rear of the auditorium acts as a partition, and more or less reflects and refracts the speaker's utterances to the audience. In the absence of the author, a paper by Mr. J. W. Gordon describing an ingenious new micrometer was taken as read.

Mr. W. H. F. Murdoch gave an exhibition and description of a friction permeameter. This is a development of one previously designed by the author. In the new form the defects of the old form are removed. Dr. J. A. Harker followed with a paper (by himself and Mr. W. F. Higgins) on the methods and apparatus used in petroleum testing. He stated that it is well known that the Abel-Pensky apparatus gives a higher result for the same oil than the Abel. The results of experiments made at the National Physical Laboratory by the authors show that large differences of temperature exist throughout the oil cup and vapour space above it at any stage during an experiment amounting to $5^{\circ} \mathrm{F}$. or more, and they are different in the different types of apparatus. The size of the test flame is also shown to be of importance. The difference of temperature in the two types is found to be due to the cover in the Abel-Pensky form containing much more metal, and therefore requiring longer to warm up.

The proceedings on Monday, September 4, began with a joint discussion with Section $G$ on mechanical flight. A special article on this discussion has appeared elsewhere in this journal, and consequently it will not be further mentioned here.

\section{Corpuscular Radiation.}

A discussion was opened by an extremely lucid and persuasive paper by Prof. W. H. Bragg on corpuscular radiation. Such radiation he defined as consisting of entities or "quanta" each moving in a straight line with uniform velocity and unchanging properties unless impressed forces cause a change. The $\alpha$ and $\beta$ rays are corpuscular, but not sound or light as ordinarily conceived. With regard to $\mathrm{X}$ (and $\gamma$ ) rays, it must be observed that the speed and direction of a $\beta$ ray produced by an X-ray depend on the quality of the X-ray and not on the nature of the atom. The energy of the $\beta$ ray, therefore, cannot come from the atom; nor yet can it be the result of the accumulation in the atom of energy extracted from many X-rays, for it can hardly be supposed that the accession of the last infinitesimal amount of energy required would determine so effectively the direction in which the $\beta$ ray is ejected. Therefore one $\mathrm{X}$-ray provides the energy for one $\beta$ ray, and vice versa, and Whiddington's results show that very little energy is lost in the transformation. Again, since the speed of the secondary $\beta$ ray is independent of the distance which the X-ray has travelled, the latter cannot diffuse its energy as it proceeds. Again, X-rays can excite $X$-rays of less, but not greater, penetrating power than themselves; and they must have arisen from $\beta$ rays of energy exceeding a certain limit, viz. that characteristic of the X-corpuscle of that substance. The spreading pulse of Stokes and the kink in a tube of force (Thomson) fail to account for these facts; only a corpuscular theory will do this. Prof. Bragg's working model of such a corpuscle is a " neutral pair.

Sir Wm. Ramsay in the ensuing discussion asked whether a similar explanation might not account for the sun remaining hot so long, assuming it only sends out radiations in the directions for which a body exists to receive them. He also directed attention to experiments of his own in which radium enclosed in a glass tube surrounded by a second vessel gradually excited radio-activity in the latter. If the outer vessel is dissolved, an active substance can be chemically precipitated. His own theory is that the $\beta$ rays shot out from the radium excite an actual chemical change in which a radio-active substance is produced.

Dr. Lindemann suggested that a crucial test between corpuscular and wave theory is supplied by the value of radiation pressure being twice as great on the corpuscular theory, but the reply came that the pressure is too small to be measured at present. Pulses of genuine delight ran through the meeting while Prof. Bragg expounded his views, and it was clear that many were impressed by the cogency of his arguments.

In a paper on the dependence of the spectrum of an element on its atomic weight, Prof. W. M. Hicks showed how to represent by formulæ the lines of a spectrum which are related to the well-known series in the same way as the second or third set of a doublet or triplet series depends on the first, or as the satellite lines of a D series depend on the more intense set. Examples were given from the sharp series of $\mathrm{Mg}, \mathrm{Ca}$, and $\mathrm{Sr}$, but the other series show similar connections. In the absence of Major E. H. Hills a paper by him on the arc spectra of certain metals in the infra-red $(\lambda 7600$ to $\lambda$ 10,000) was taken as read. This paper details the results of measurements made on photographic plates coated with a collodio-bromide emulsion, as originally used by Abney in 1880 . Measurements by others have usually been made with a thermopile or bolometer.

Dr. Harker read a paper, on behalf of Mr. H. C. Green-

No. 2 I89, VOL. 87] 
wood, outlining an investigation on specific heats of metals, especially in the neighbourhood of their melting points, and determinations of the latent heat of fusion. The metals dealt with in this paper are aluminium and zinc.

The first paper read on Wednesday was by $\mathrm{Mr}$. H. Davies, on the laws of solution. Mr. Davies showed how the formula of Rudolphi, which had been suggested to represent the cases where Ostwald's law of dilution breaks down, can be placed upon a theoretical basis. Hitherto it has been held as a purely empirical formula (see in relation to this subject the address of the president of Section B, Nature, p. 297). Mr. Davies's contribution is of great importance to the physical chemist, inasmuch as it elucidates "the outstanding practical problem in the domain of electrolytic solutions."

Prof. P. V. Bevan contributed a paper on anomalous dispersion and solar phenomena. If light from a nonuniform source, such as an arc light, be sent through a tube containing non-homogeneous vapour of a metal which can show anomalous dispersion, and then in image of the source be focussed on the slit of a spectroscipe, an apparent double reversal of certain lines may be produced. An explanation is given based upon the bright images of the two poles of the arc being formed on a darker background of light which has come from elsewhere through the vapour; the essence of the explanation is that the source shall be non-uniform. It was suggested that such apparent reversals might have a bearing upon solar phenomena.

\section{LINKS WITH THE PAST IN THE PLANT WORLD. ${ }^{1}$}

UNDER the heading "Links with the Past," several letters were published in The Times rather more than a year ago in which the writers gave instances of human longevity, showing how in certain cases a chain of a very few individuals suffices to connect the present with a comparatively remote past. One writer, for example, said that his grandmother, who died about forty years ago, used to boast that her grandfather was twelve years old when Charles I. was beheaded. Striking as such instances are when applied to man, on the other hand they serve to illustrate the relative insignificance of the length of time represented by human lives as contrasted with the duration of many forest trees. It is probably not an exaggeration to say that a single oak tree may form a link between the present day and the Norman Conquest; a very short series of ancestors suffices to carry us back to the days when the progress of the Roman invaders was seriously impeded by dense forests, which have long since disappeared, and farther back to the age of Neolithic man, whose flint implements are occasionally met with in the submerged forests round our coasts.

It would be interesting, if time permitted and my knowledge were adequate, to consider some of our forest trees from the point of view of their past history. The great majority of existing woods in Britain are the result of cultivation, and do not come within our purview in dealing with links with a remote past. Moreover, many of our familiar British trees, such as the common elm, the lime, the chestnuts, and others, have no claim to be classed as native, but were introduced in Roman or post-Roman days. In a few places in Inverness-shire and Perthshire patches of primæval forest survive; one of them is represented by the group of Scots pines growing in the Black Wood of Rannoch, in north-west Perthshire. Excavations in the Scotch peat-moors have revealed a succession of forests and wet moorland; in some places, e.g. in the Outer Hebrides, these buried forests occur in districts which are now almost treeless. As shown in sections recently published by Dr. Lewis, a cutting through 20 or 30 feet of Highland peat gives us an epitome of the changing physical and climatic conditions from the close of the Glacial period to the present day. The remains of Arctic willows, the crowberry (Empetrum nigrum), and other northern species immediately above the glacial deposits testify to the influence of the Ice age. The Arctic plants are succeeded by vegetation indicative of a milder

1 Evening discourse delivered before the British Association at Portsmouth on September 4 by Prof. A. C. Seward, F.R.S. climate; layers of bog-moss and stumps of pines point to an alternation of forests and wet moorland.

The spruce fir, one of our best known trees, affords an example of a species which was once a native, but is no longer found in a wild state. The cone of the spruce fir shown on the screen was recently recorded by $\mathrm{Mr}$. and Mrs. Clement Reid, together with the seeds and fruits of many other plants, from deposits on the Norfolk and Suffolk coast which were formed shortly before the Glacial period. The plants of this pre-glacial flora indicate a temperate climate, but the nearer approach of more severe conditions is shown by the Arctic willow and dwar birch which have been found in beds next above those containing the spruce fir.

The occurrence of the Glacial period is a fact of primary importance in relation to the antiquity of the present flora of this country. We know that Britain in comparatively recent times, speaking geologically, was in very much the same condition as Greenland is to-day. Over nearly the whole of Scotland, Ireland, Wales, and England, with the exception of a narrow strip in the south, there is clear evidence of ice action on a large scale and of the presence of ice-sheets and local glaciers. Under these Arctic conditions it can hardly be doubted that only a very small proportion of the vegetation could survive. Opinions differ as to the extent to which the Ice age proved fatal to the pre-glacial flora, but it is perhaps not too much to say that the present flora, as a whole, is of post-glacial date. The vegetation which grew in this part of Europe before the Glacial period reached its maximum must have been in great measure destroyed or driven south beyond the British area. The important point is that what we call our native flowering plants may safely be described in general terms as immigrants from other lands, aided, it may be, by land-connections across the North Sea and English Channel.

Special interest attaches to a few plants which occur in the west and south of Ireland, and to a less extent in Cornwall and elsewhere in the south-west of England. In Connemara in the west of Ireland, where hard frosts are unknown and winter snows rare, there are three kinds of heath, St. Dabeoc's heath (Dabeocia polifolia), the Mediterranean heath (Erica mediterranea), and Erica Mackaii, which are not found elsewhere in the British Isles or in the whole of northern Europe, but reappear in the Pyrenees. The London pride (Saxifraga umbrosa), another Pyrenean species, grows on the south and west coasts of Ireland from Waterford to Donegal. Arbutus Unedo (the strawberry tree), scattered through the Killarney woods, has a wide distribution in the Mediterranean region, its nearest continental station being in the south-west of France.

The presence of this small group of Mediterranean and Lusitanian plants in Ireland has long been a puzzle to naturalists. A few years ago I came across a solution to the problem of these southern plants in Ireland in a collection of stories entitled "A Child's Book of Saints." An Irish monk, Bresal, was sent to teach the brethren in a Spanish monastery the music of Irish choirs. In later years he longed for a sight of his native land, to which he at length returned: his thoughts reverted to Spain, and he saw once more the little white flowers of the Saxifrage and the strawberry tree from which he had gathered the orange-scarlet berries. With heavenly vision the prior of the Spanish monastery, seeing Bresal gazing at the flowers of Spain, commanded them to go and make real his dream. Thus, to gladden the heart of the monk, were these southern plants miraculously introduced into Ireland.

One view is that Arbutus and its companions are entitled to be regarded as a very old section of the British flora, survivals from a time, the so-called Tertiary period, when the climate was much milder than it is to-day. It is believed by some authorities that these plants migrated from Portugal to Ireland long before the Glacial period, and by means of a land-bridge, which afterwards sank below the waters of the Atlantic Ocean. This explanation is open to criticism; even granting the former existence of a land-connection, it is difficult to believe that the strawberry tree, which is restricted to one of the warmest districts in the British Isles, could have survived the rigours of the Glacial period. Moreover, as Mr. Clement 